$\underline{\text { Preprint typeset in JHEP style - HYPER VERSION }}$

\title{
Primordial SdS universe from a 5D vacuum: scalar field fluctuations on Schwarzschild and Hubble horizons
}

\author{
José Edgar Madriz Aguilar, \\ Instituto de Física de la Universidad de Guanajuato, C.P. 37150, León Guanajuato, México. \\ E-mail: jemadriz@fisica.ugto.mx \\ Mauricio Bellini \\ Departamento de Física, Facultad de Ciencias Exactas y Naturales, Universidad Nacional de \\ Mar del Plata, Funes 3350, C.P. 7600, Mar del Plata, Argentina. \\ Instituto de Investigaciones en Física, Consejo Nacional de Investigaciones Científicas y \\ Técnicas (CONICET), Argentina. \\ E-mail: mbellini@mdp.edu.ar, mbellini@conicet.gov.ar
}

\begin{abstract}
We study scalar field fluctuations of the inflaton field in an early inflationary universe on an effective 4D Schwarzschild-de Sitter (SdS) metric, which is obtained after make a planar coordinate transformation on a 5D Ricci-flat Schwarzschild-de Sitter (SdS) static metric. We obtain the important result that the spectrum of fluctuations at zeroth order is independent of the scalar field mass $M$ on Schwarzschild scales, while on cosmological scales it exhibits a mass dependence. However, in the first-order expansion, the spectrum depends of the inflaton mass and the amplitude is linear with the Black-Hole (BH) mass $m$.
\end{abstract}

KEYWORDS: physics of the early universe, cosmology with extra dimensions. 


\section{Contents}

1. Introduction 1

2. The 5D scalar field equation of motion 3

2.1 The $5 \mathrm{D}$ dynamics of the scalar field 3

2.2 The $4 \mathrm{D}$ induced field equation 1

3. The expansion of the induced scalar field $\bar{\varphi}$

3.1 The zeroth order expansion for the induced scalar field 6

3.1.1 Zeroth order: power spectrum on cosmological scales

3.1.2 Zeroth order: power spectrum on Schwarzschild scales 8

3.2 First order expansion of the induced scalar field 8

3.2.1 First order: power spectrum on cosmological scales 10

3.2.2 First order: power spectrum on small scales 11

4. Final comments 12

\section{Introduction}

The cosmic microwave background that we observe today is almost isotropic. The background temperature is about $2.7 \mathrm{~K}$ with a tiny fluctuation at a level of about $10^{-5} \mathrm{k}$. This is consistent with measurements of matter structures in the universe at cosmological scales, where the universe is almost homogeneous. However, it is well known that the mass spectrum on astrophysical and galactic scales has a negative index which decreases with the observed scale. The studies developed in the last years have shown that on astrophysical scales the power spectrum of galaxies and clusters of galaxies can be satisfactorily expressed by a power law with an index between -1.9 and -1.5 [1]. On larger scales the spectrum turns over reaching a maximum on scales of $(100-150) h^{-1} M p c$. For our purposes we shall assume that the evolution of the structure in the universe is only due to gravity.

Astrophysical and Cosmological systems have been also theoretically investigated, in the context of theories of gravity in more than four dimensions (4D). In theories with five dimensions (5D), models fit the observations due in much because they smoothly embed 4D solutions in 5D solutions [2]. Since the M-theory and quantum geometry have appeared as promising candidates for a quantum theory of gravity [3], the idea that our universe is a $4 \mathrm{D}$ space-time embedded in a higher dimensional manifold with large extra dimensions, has been a topic of increased interest in several branches of physics, and in particular, in cosmology. This idea that goes back to the works of Nordström [4] and the original version of the Kaluza-Klein theory [5], has generated a new kind of cosmological models, including the type of quintessential and dark energy models.

In particular, theories regarding just one extra dimension have become quite popular in the scientific community. Two proposals that have been subject of great research are the brane theory [6] and the induced matter (IM) theory of gravity [7]. Although brane theory and IM theory have 
different physical motivations for the introduction of a large extra dimension, they are equivalent each other [8]. They both predict identical non-local and local high energy corrections to general relativity in $4 \mathrm{D}$, and usual matter in $4 \mathrm{D}$ is a consequence of the metric dependence on the fifth extra coordinate 8$]$.

In this letter we have particular interest in the ideas of the IM theory. A success of this theory is that classical properties of matter fields in $4 \mathrm{D}$ can be given a geometrical interpretation if we assume a $5 \mathrm{D}$ apparent vacuum, defined by the condition: ${ }^{(5)} R_{a b}=0$. Matter fields in $4 \mathrm{D}$ are described by an effective 4D energy-momentum tensor purely geometrical in origin [9]. In particular, according to [2] the mass of classical particles in 4D is directly related with the existence of the fifth extra coordinate. In this manner in the IM theory the mass of classical particles has a geometrical origin and this feature makes this theory attractive from the fundamentalist point of view. Furthermore, the fact that any energy-momentum tensor can be geometrically induced in this manner, is hardly supported by the Campbell-Magaard theorem and their extensions 10 . A particular and very interesting 1-body solution of the 5D field equations of the IM theory is the 5D Ricci-flat Schwarzschild-de Sitter (SdS) black-hole, which contains an induced cosmological constant 11. This solution can be embedded into a brane world by using a conformal factor 12] and constitutes a 5D Ricci-flat SdS black string space. This black string space is the Randall and Sundrum [13] 2-brane model. We have recently shown in 14] that this 5D SdS BH solution give us in $4 \mathrm{D}$ ordinary gravitational solutions on small (planetary and astrophysical) scales, but repulsive (anti-gravitational) forces on very large (cosmological) scales. We called the effective 4D gravitational theory derived from this scenario, an extended version of general relativity. This behavior of gravity on different length scales derived from this 5D framework, leave us to put on the desk the question weather repulsive gravity can be considered as a candidate for explaining the present accelerated expansion of the universe. However, as it is well known in the literature, inflation is another period of accelerated expansion that the universe has passed through. So it makes interesting to ask about if inflation still works in this extended version of general relativity. In order to address the former question, in this letter we define a free scalar field on a 5D geometrical background, on which the $5 \mathrm{D}$ metric is expressed in planar coordinates in order to consider in $4 \mathrm{D}$, a black hole in an inflationary scenario. The original massless $5 \mathrm{D}$ scalar field can be seen in $4 \mathrm{D}$ as a massive scalar field, that we identify with the inflaton field. The fifth extra coordinate is responsible for the 5D massless scalar field to acquire mass in 4D. This way using some ideas of the IM theory we can give a geometrical origin for the inflaton mass in $4 \mathrm{D}$, and this is one contribution of the extra coordinate in $4 \mathrm{D}$. Our goal in this letter is to study the evolution of quantum fluctuations during inflation on cosmological scales, on which gravitational effects are repulsive, and also on very short scales (which are close to the Schwarzschild scale), where gravity manifests itself as attractive.

The letter is organized as follows. In section I we give a brief introduction. In section II we obtain the dynamical equations for the inflaton scalar field in $5 \mathrm{D}$ and $4 \mathrm{D}$. We then proceed to section III, divided in several subsections, in which we calculate the inflaton quantum fluctuations on short (Schwarzschild) scales and large (cosmological) scales. We have implemented an expansion for the inflaton field in powers of a small parameter that arises naturally from the model, so we calculate the power spectrum on short and large scales at zeroth and first orders in the expansion only. Finally in section IV, we give some final comments. 


\section{The 5D scalar field equation of motion}

We consider the 5D Ricci-flat metric [14]

$$
d S_{5}^{2}=\left(\frac{\psi}{\psi_{0}}\right)^{2}\left[c^{2} f(R) d T^{2}-\frac{d R^{2}}{f(R)}-R^{2}\left(d \theta^{2}+\sin ^{2} \theta d \phi^{2}\right)\right]-d \psi^{2},
$$

where $f(R)=1-\left(2 G \zeta \psi_{0} / R c^{2}\right)-\left(R / \psi_{0}\right)^{2}$ is a dimensionless function, $\{T, R, \theta, \phi\}$ are the usual local spacetime spherical coordinates employed in general relativity and $\psi$ is the space-like extra dimension. Following the approach of the IM theory, $\psi$ is here on considered as non-compact. This metric is a $5 \mathrm{D}$ extension of the $4 \mathrm{D}$ SdS metric. In this line element $\psi$ and $R$ have length units, $\theta$ and $\phi$ are angular coordinates, $T$ is a time-like coordinate, $c$ denotes the speed of light, $\psi_{0}$ is an arbitrary constant with length units and the constant parameter $\zeta$ has units of $($ mass $)(\text { length })^{-1}$. As we can see, this metric is written on a static chart coordinate, so in order to get this metric written on a dynamical chart coordinate $\{t, r, \theta, \phi\}$, let us to use the coordinate transformation given by 15$]$

$$
R=a r\left[1+\frac{G \zeta \psi_{0}}{2 a r}\right]^{2}, \quad T=t+H \int^{r} d R \frac{R}{f(R)}\left(1-\frac{2 G \zeta \psi_{0}}{R}\right)^{-1 / 2}, \psi=\psi,
$$

being $a(t)=e^{H t}$ the scale factor, and $H$ the Hubble constant. Thus the line element (2.1) can be written in terms of the conformal time $\tau$ as

$$
d S_{5}^{2}=\left(\frac{\psi}{\psi_{0}}\right)^{2}\left[F(\tau, r) d \tau^{2}-J(\tau, r)\left(d r^{2}+r^{2}\left(d \theta^{2}+\sin ^{2} \theta d \phi^{2}\right)\right)\right]-d \psi^{2}
$$

where the metric functions $F(\tau, r)$ and $J(\tau, r)$ are given by

$$
F(\tau, r)=a^{2}(\tau)\left[1-\frac{G \zeta \psi_{0}}{2 a(\tau) r}\right]^{2}\left[1+\frac{G \zeta \psi_{0}}{2 a(\tau) r}\right]^{-2}, \quad J(\tau, r)=a^{2}(\tau)\left[1+\frac{G \zeta \psi_{0}}{2 a(\tau) r}\right]^{4},
$$

with $d \tau=a^{-1}(\tau) d t$ and $a(\tau)=-1 /(H \tau)$, so that the Hubble parameter is a constant given by

$$
H=a^{-2} \frac{d a}{d \tau} .
$$

As it was shown in [14, for certain values of $\zeta$ and $\psi_{0}$ the metric in (2.1) has two natural horizons. The inner horizon is the analogous of the Schwarzschild horizon and the external one is the analogous of the Hubble horizon. In the metric (2.3), these horizons can in principle be expressed in terms of the new dynamical coordinates.

\subsection{The 5D dynamics of the scalar field}

Now we consider a 5D massless scalar field which is free of any interactions. The dynamics is given by the Klein-Gordon type field equation

$$
\frac{1}{\sqrt{\left|g_{5}\right|}} \frac{\partial}{\partial y^{a}}\left[\sqrt{\left|g_{5}\right|} g^{a b} \varphi_{, b}\right]=0
$$

where $\sqrt{\left|g_{5}\right|}=\left(\psi / \psi_{0}\right)^{4} F^{1 / 2} J^{3 / 2} r^{2} \sin \theta$ is the determinant of the covariant metric tensor $g_{a b}$. On the metric (2.3), the equation (2.6) becomes

$$
\frac{1}{\sqrt{F J^{3}}} \frac{\partial}{\partial \tau}\left(\sqrt{\frac{J^{3}}{F}} \frac{\partial \varphi}{\partial \tau}\right)-\frac{1}{2}\left(\frac{1}{F J} \frac{\partial F}{\partial r}+\frac{1}{J^{2}} \frac{\partial J}{\partial r}\right) \frac{\partial \varphi}{\partial r}-\frac{1}{J} \nabla^{2} \varphi-\left(\frac{\psi}{\psi_{0}}\right)^{-2} \frac{\partial}{\partial \psi}\left[\left(\frac{\psi}{\psi_{0}}\right)^{4} \frac{\partial \varphi}{\partial \psi}\right]=0,
$$


where

$$
\nabla^{2} \equiv \frac{1}{r^{2}} \frac{\partial}{\partial r}\left(r^{2} \frac{\partial}{\partial r}\right)+\frac{1}{r^{2} \sin \theta} \frac{\partial}{\partial \theta}\left(\sin \theta \frac{\partial}{\partial \theta}\right)+\frac{1}{r^{2} \sin ^{2} \theta} \frac{\partial^{2}}{\partial \phi^{2}}
$$

is the 3D Laplacian in spherical coordinates.

Assuming that $\varphi(\tau, r, \theta, \phi, \psi)$ can be separated in the form

$$
\varphi(\tau, r, \theta, \phi, \psi) \sim \Phi(\tau, r) G(\theta, \phi) \Omega(\psi),
$$

the expression (2.7) leaves to

$$
\begin{aligned}
& \left(\frac{\psi}{\psi_{0}}\right)^{-2} \frac{d}{d \psi}\left[\left(\frac{\psi}{\psi_{0}}\right)^{4} \frac{d \Omega}{d \psi}\right]+M^{2} \Omega=0, \\
& \frac{1}{\sqrt{F J}} \frac{\partial}{\partial \tau}\left(\sqrt{\frac{J^{3}}{F}} \frac{\partial \Phi}{\partial \tau}\right)-\frac{1}{2}\left(\frac{1}{F} \frac{\partial F}{\partial r}+\frac{1}{J} \frac{\partial J}{\partial r}\right) \frac{\partial \Phi}{\partial r}-\frac{1}{r^{2}} \frac{\partial}{\partial r}\left(r^{2} \frac{\partial \Phi}{\partial r}\right) \\
& -\left(\frac{l(l+1)}{r^{2}}-M^{2} J\right) \Phi=0,
\end{aligned}
$$

where $M$ is a separation constant with mass units and $l$ is an integer parameter. In deriving (2.11) we have separated out the angular part of $\varphi$.

\subsection{The $4 \mathrm{D}$ induced field equation}

Assuming that the 5D spacetime can be foliated by a family of hypersurfaces $\Sigma: \psi=\psi_{0}$, from the metric (2.3) we obtain that the $4 \mathrm{D}$ induced metric on every leaf $\Sigma: \psi=\psi_{0}$ is given by

$$
d S_{4}^{2}=F(\tau, r) d \tau^{2}-J(\tau, r)\left[d r^{2}+r^{2}\left(d \theta^{2}+\sin ^{2} \theta d \phi^{2}\right)\right],
$$

where the metric functions $F(\tau, r)$ and $J(\tau, r)$ can be now written in terms of the physical mass $m=\zeta \psi_{0}$ (introduced by the first time in [14]), in the form

$$
F(\tau, r)=a^{2}(\tau)\left[1-\frac{G m}{2 a(\tau) r}\right]^{2}\left[1+\frac{G m}{2 a(\tau) r}\right]^{-2}, \quad J(\tau, r)=a^{2}(\tau)\left[1+\frac{G m}{2 a(\tau) r}\right]^{4},
$$

valid for $r>G m /(2 a)$. The induced metric (2.12) describes a black hole in an expanding universe, where the expansion is driven by a kind of cosmological constant, whose value in general depends of the value of $\psi_{0}$, which is related to the Hubble constant: $\psi_{0}=1 / H$. From the equations (2.7) and (2.10), the $4 \mathrm{D}$ induced field equation on $\Sigma$, reads

$$
\frac{1}{\sqrt{F J^{3}}} \frac{\partial}{\partial \tau}\left[\sqrt{\frac{J^{3}}{F}} \frac{\partial \bar{\varphi}}{\partial \tau}\right]-\frac{1}{2}\left(\frac{1}{F J} \frac{\partial F}{\partial r}+\frac{1}{J^{2}} \frac{\partial J}{\partial r}\right) \frac{\partial \bar{\varphi}}{\partial r}-\frac{1}{J} \nabla^{2} \bar{\varphi}+M^{2} \bar{\varphi}=0,
$$

where $\bar{\varphi}(\tau, r, \theta, \phi)=\varphi\left(\tau, r, \theta, \phi, \psi_{0}\right)$ is the effective scalar field induced on the generic hypersurface $\Sigma$. Here, $M$ plays the role of the mass of the inflaton field on the 4D brane (2.12), such that the effective $4 \mathrm{D}$ equation of motion for $\bar{\varphi}$ reads: $\left[{ }^{(4)} \square+M^{2}\right] \bar{\varphi}=0$. This result was previously obtained in a different manner using the fact that the 5D linear momentum $P^{a}=M u^{a}$ is conserved on a 5D Ricci flat spacetime, so that $P_{; a}^{a}=0$ 16. Hence, as in other 5D inflationary models, the 4D mass of the inflaton field is induced geometrically through the foliation $\psi=\psi_{0}$, because on the 5D metric (2.7) the equation of motion for $\varphi(\tau, r, \theta \phi)$ is ${ }^{(5)} \square \varphi(\tau, r, \theta, \phi)=0$, so that the field $\varphi$ can be viewed in the spacetime (2.7) as a free and massless scalar field. Furthermore, the extra dimension is related to the $\mathrm{BH}$ mass $m: \psi_{0}=m / \zeta=c^{2} / H$ (we use natural units $c=\hbar=1$ ). 
On the other hand, according to 14] the length scale that separates regions on which gravity changes from attractive to repulsive, is the gravitational-antigravitational radius, which in the coordinates $(T, R)$ is given by

$$
R_{g a}=\left(G m \psi_{0}^{2}\right)^{1 / 3} .
$$

However, due to the coordinate transformation (2.2), in the new coordinates $(\tau, r)$ this radius must obey the relation

$$
R_{g a}=a(\tau) r_{g a}\left[1+\frac{G m}{2 a(\tau) r_{g a}}\right]^{2}
$$

where $r_{g a}$ is denoting the gravitational-antigravitational radius in the new coordinates. Solving this equation for $r_{g a}$ we obtain

$$
r_{g a}=\frac{1}{2 a(\tau)}\left[R_{g a}-G m \pm \sqrt{R_{g a}^{2}-2 G m R_{g a}}\right]
$$

where the solution with the minus sign is not a physical solution. Moreover, in order to $r_{g a}$ to be a real value quantity, we require the condition $R_{g a}^{2}-2 G m R_{g a} \geq 0$ to be hold. This condition can be rewritten in the form

$$
R_{g a} \geq 2 G m .
$$

Inserting (2.15) in (2.18), we obtain the restriction

$$
m^{2} \leq \frac{\psi_{0}^{2}}{8 G^{2}}
$$

If we consider the foliation $\psi_{0}=c^{2} / H$ and the fact that for $c=\hbar=1$ the Newtonian constant is $G=M_{p}^{-2}$, the condition (2.19) yields

$$
\epsilon=\frac{m H}{M_{p}^{2}} \leq \frac{1}{2 \sqrt{2}} \simeq 0.353553
$$

and hence the fifth coordinate must be restricted to the following condition:

$$
\psi_{0} \geq \frac{2 \sqrt{2} m}{M_{p}^{2}} .
$$

This condition allow us to consider objects whose mass satisfies the parameter $\epsilon=G m H$ to be a small parameter. The same restriction has been used in [18] with different motivation, without affecting the range of validity of the dynamical coordinates.

\section{The expansion of the induced scalar field $\bar{\varphi}$}

We expand the induced scalar field $\bar{\varphi}$ as

$$
\bar{\varphi}(\vec{r}, \tau)=\int_{0}^{\infty} d k \sum_{l m}\left[a_{k l m} \bar{\Phi}_{k l m}(\vec{r}, \tau)+a_{k l m}^{\dagger} \bar{\Phi}_{k l m}^{*}(\vec{r}, \tau)\right]
$$

where

$$
\bar{\Phi}_{k l m}(\vec{r}, \tau)=k^{2} j_{l}(k r) \bar{\Phi}_{k l}(\tau) Y_{l m}(\theta, \phi),
$$

where $Y_{l m}(\theta, \phi)$ are the spherical harmonics, $j_{l}(k r)$ are the spherical Bessel functions and the annihilation and creation operators obey the algebra

$$
\left[a_{k l m}, a_{k^{\prime} l^{\prime} m^{\prime}}^{\dagger}\right]=\delta\left(k-k^{\prime}\right) \delta_{l l^{\prime}} \delta_{m m^{\prime}}, \quad\left[a_{k l m}, a_{k^{\prime} l^{\prime} m^{\prime}}\right]=\left[a_{k l m}^{\dagger}, a_{k^{\prime} l^{\prime} m^{\prime}}^{\dagger}\right]=0 .
$$


Hence, once we take into account the addition theorem for spherical harmonics, we obtain for the mean squared fluctuations

$$
\left\langle 0\left|\bar{\varphi}^{2}(\vec{r}, \tau)\right| 0\right\rangle=\int_{0}^{\infty} \frac{d k}{k} \sum_{l} \frac{2 l+1}{4 \pi} k^{5} j_{l}^{2}(k r)\left|\bar{\Phi}_{k l}(\tau)\right|^{2} .
$$

Now, if we assume that $\bar{\varphi}(\tau, r, \theta, \phi)=\bar{\Phi}(\tau, r) \bar{G}(\theta, \phi)$, then the equation for $\bar{\Phi}(r, \tau)$ on the hypersurface $\Sigma$ can be written as

$$
\begin{aligned}
\frac{\partial^{2} \bar{\Phi}_{l}}{\partial \tau^{2}} & -\frac{2}{\tau} \frac{\partial \bar{\Phi}_{l}}{\partial \tau}-\frac{2}{r} \frac{\partial \bar{\Phi}_{l}}{\partial r}-\frac{\partial^{2} \bar{\Phi}_{l}}{\partial r^{2}}-\left[\frac{l(l+1)}{r^{2}}-M^{2}\right] \bar{\Phi}_{l} \\
& =\left(1-\frac{J}{F}\right) \frac{\partial^{2} \bar{\Phi}_{l}}{\partial \tau^{2}}-\left[\frac{2}{\tau}+\frac{1}{\sqrt{F J}} \frac{\partial}{\partial \tau}\left(\frac{J^{3}}{F}\right)^{1 / 2}\right] \frac{\partial \bar{\Phi}_{l}}{\partial \tau} \\
& -M^{2}(J-1) \bar{\Phi}_{l}+\frac{1}{2}\left(\frac{1}{F} \frac{\partial F_{l}}{\partial r}+\frac{1}{J} \frac{\partial J}{\partial r}\right) \frac{\partial \bar{\Phi}_{l}}{\partial r}
\end{aligned}
$$

Next,using the fact that $\epsilon$ is a small parameter as indicated by 2.20), we propose the following expansion for $\bar{\Phi}_{l}$ in orders of $\epsilon$ :

$$
\bar{\Phi}_{l}(r, \tau)=\bar{\Phi}_{l}^{(0)}+\bar{\Phi}_{l}^{(1)}+\bar{\Phi}_{l}^{(2)}+\ldots
$$

If we expand the right hand side of the equation (3.5) as powers of $\epsilon$ 18, we obtain

$$
\begin{aligned}
& -8\left(\frac{\epsilon \tau}{2 r}\right)\left[\frac{\partial^{2} \bar{\Phi}_{l}^{(0)}}{\partial \tau^{2}}-\frac{1}{\tau} \frac{\partial \bar{\Phi}_{l}^{(0)}}{\partial \tau}-\frac{M^{2}}{2 H^{2} \tau^{2}} \bar{\Phi}_{l}^{(0)}\right] \\
& -30\left(\frac{\epsilon \tau}{2 r}\right)^{2}\left[\frac{\partial^{2} \bar{\Phi}_{l}^{(1)}}{\partial \tau^{2}}-\frac{1}{15} \frac{1}{\tau} \frac{\partial \bar{\Phi}_{l}^{(1)}}{\partial \bar{\Phi}}-\frac{M^{2}}{5 H^{2} \tau^{2}} \bar{\Phi}_{l}^{(1)}\right]+\ldots
\end{aligned}
$$

Thus, we are now able to calculate solutions for $\bar{\Phi}_{l}(r, \tau)$ at zeroth and first orders in the expansion. Higher orders require numerical analysis.

Finally, the spectrum for the squared fluctuations (3.4) can be written using the expansion (3.6) in the following manner

$$
\begin{aligned}
\mathcal{P}_{k}(\tau) & =\sum_{l} \frac{(2 l+1)}{4 \pi} k^{5} j_{l}^{2}(k r)\left[\bar{\Phi}_{k l}^{(0)}+\bar{\Phi}_{k l}^{(1)}+\ldots\right]\left[\left(\bar{\Phi}_{k l}^{(0)}\right)^{*}+\left(\bar{\Phi}_{k l}^{(1)}\right)^{*}+\ldots\right] \\
& =\frac{k^{3}}{2 \pi^{2}}\left|\bar{\Phi}_{k l}^{(0)}\right|^{2}+\frac{H^{2}}{4 \pi^{2}} \epsilon \sum_{l=1}^{\infty}(2 l+1) j_{l}^{2}(k r) \Delta_{k l}^{(1)}+\ldots,
\end{aligned}
$$

such that

$$
\left.\Delta_{k l}^{(1)}=\left(\frac{4 \pi^{2}}{H^{2} \epsilon}\right) \frac{k^{5}}{4 \pi} \mid \bar{\Phi}_{k l}^{(0)}\left(\bar{\Phi}_{k l}^{(1)}\right)^{*}+\bar{\Phi}_{k l}^{(1)}\left(\bar{\Phi}_{k l}^{(0)}\right)^{*}\right]=\frac{2 \pi}{H^{2} \epsilon} k^{5} \operatorname{Re}\left[\bar{\Phi}_{k l}^{(1)}\left(\bar{\Phi}_{k l}^{(0)}\right)^{*}\right]
$$

In the following subsections we shall study the spectrum at first-order, as expanded in (3.8). We are interested mainly in the two limit cases; the Hubble and the Schwarzschild horizons.

\subsection{The zeroth order expansion for the induced scalar field}

Using the equations (3.5), (3.6) and (3.7), we obtain that the dynamics for a massive scalar field in a spatially homogeneous de Sitter case is described by

$$
\frac{\partial^{2} \bar{\Phi}_{l}^{(0)}}{\partial \tau^{2}}-\frac{2}{\tau} \frac{\partial \bar{\Phi}_{l}^{(0)}}{\partial \tau}-\frac{2}{r} \frac{\partial \bar{\Phi}_{l}^{(0)}}{\partial r}-\frac{\partial^{2} \bar{\Phi}_{l}^{(0)}}{\partial r^{2}}-\left[\frac{l(l+1)}{r^{2}}-M^{2} a^{2}(\tau)\right] \bar{\Phi}_{l}^{(0)}=0,
$$


where the last term corresponds with the induced mass of the scalar field. If we use the Bessel transform

$$
\bar{\Phi}_{l}^{(0)}(r, \tau)=\int_{0}^{\infty} d k k^{2} j_{l}(k r) \bar{\Phi}_{k l}^{(0)}(\tau),
$$

we obtain the zeroth order dynamics for the modes $\bar{\Phi}_{k l}^{(0)}$

$$
\frac{\partial^{2} \bar{\Phi}_{k l}^{(0)}}{\partial \tau^{2}}-\frac{2}{\tau} \frac{\partial \bar{\Phi}_{k l}^{(0)}}{\partial \tau}+\left(k^{2}+\frac{M^{2}}{H^{2} \tau^{2}}\right) \bar{\Phi}_{k l}^{(0)}=0 .
$$

If we use the Bunch-Davies vacuum, we obtain the normalized modes solution

$$
\bar{\Phi}_{k l}^{(0)}=A_{1}(-\tau)^{3 / 2} \mathcal{H}_{\nu}^{(1)}[-k \tau]+A_{2}(-\tau)^{3 / 2} \mathcal{H}_{\nu}^{(2)}[-k \tau],
$$

where $\mathcal{H}_{\nu}^{(1,2)}$ are respectively the first and second kind Hankel functions with $\nu^{2}=\frac{9}{4}-\frac{M^{2}}{H^{2}}$. The normalization constants are

$$
A_{2}=-\frac{\sqrt{\pi} H}{2} e^{-i \nu \pi / 2}, \quad A_{1}=0
$$

If we take into account that at zero order $l$ can take only the value $l=0$, we obtain that the spectrum of the fluctuations without sources is

$$
\mathcal{P}_{k l}^{(0)}(\tau)=\frac{k^{3}}{2 \pi^{2}}\left|\bar{\Phi}_{k l}^{(0)}\right|^{2}=\frac{H^{2}}{\pi}\left(\frac{-k \tau}{2}\right)^{3} \mathcal{H}_{\nu}^{(2)}[-k \tau] \mathcal{H}_{\nu}^{(1)}[-k \tau]
$$

where $k$ given by

$$
k=\frac{2 \pi}{a(\tau) r}\left[1+\frac{G m}{2 a(\tau) r}\right]^{-2},
$$

is the wave number on a physical frame.

\subsubsection{Zeroth order: power spectrum on cosmological scales}

On cosmological scales we obtain that the following inequality is fulfilled

$$
\frac{G m}{2 a(\tau) r_{H}} \ll 1
$$

so that the wavenumber of fluctuations at the horizon entry will be

$$
k_{H} \simeq \frac{2 \pi}{a(\tau) r_{H}}=-\frac{2 \pi H \tau}{r_{H}}
$$

where we have made use of the fact that $a(\tau)=-1 /(H \tau)$, with $\tau \leq 0$. Furthermore, at the end of inflation $\tau \rightarrow 0$, so that $\mathcal{H}_{\nu}^{(2)}[-k \tau] \simeq \frac{-i}{\pi} \Gamma(\nu)(-k \tau / 2)^{-\nu}$. The power spectrum on scales close to the Hubble horizon is

$$
\left.\mathcal{P}_{k l}^{(0)}(\tau)\right|_{H}=\frac{k^{3}}{2 \pi^{2}}\left|\bar{\Phi}_{k l}^{(0)}\right|_{H}^{2} \simeq\left(\frac{\pi H \tau^{2}}{r_{H}}\right)^{3-2 \nu} \frac{\Gamma^{2}(\nu) H^{2}}{\pi^{3}}
$$

which depends on the mass of the inflaton field $M$, because $\nu^{2}=\frac{9}{4}-\frac{M^{2}}{H^{2}}$. For a nearly scale invariant power spectrum: $\nu \simeq 3 / 2$, we obtain

$$
\left.\mathcal{P}_{k l}^{(0)}(\tau)\right|_{H, \nu \simeq 3 / 2} \simeq \frac{H^{2}}{4 \pi^{2}}
$$

which is the standard result for quantum fluctuations. 


\subsubsection{Zeroth order: power spectrum on Schwarzschild scales}

Another interesting limit case is the Schwarzschild scale. On these scales the following inequality is fulfilled

$$
\frac{G m}{2 a(\tau) r_{S c h}} \simeq 1,
$$

and the wavenumber of the scalar field fluctuations, when they enter to the Schwarzschild horizon, is given by

$$
k_{S c h} \simeq \frac{8 \pi a(\tau) r_{S c h}}{(G m)^{2}}=-\frac{8 \pi r_{S c h}}{H \tau(G m)^{2}},
$$

that increases linearly with the scale factor $a(\tau)$, so that $k_{S c h} \tau \gg 1$, and the power of the spectrum at the end of inflation related to the scalar field fluctuations at zero order, is

$$
\left.\mathcal{P}_{k l}^{(0)}(0)\right|_{S c h} \simeq\left(\frac{2}{\sqrt{2}} \frac{r_{S c h}}{G m}\right)^{2} \simeq \frac{(H \tau)^{2}}{\pi},
$$

where we have used the expression (3.21). Notice that the spectrum decreases dramatically as $a^{-2}$ and tends to zero at the end of inflation (i.e., for $\tau \rightarrow 0$ ). A very important fact is that the spectrum is related to the value of the fifth coordinate (we remember that we are using natural

units): $\left.\mathcal{P}_{k l}^{(0)}(0)\right|_{S c h} \simeq \frac{\tau^{2}}{\pi \psi_{0}^{2}}$, which also is related to the mass, $m$, of the $\mathrm{BH}$ through the condition (2.21).

\subsection{First order expansion of the induced scalar field}

The first order expansion for $\bar{\Phi}$ satisfies

$$
\begin{aligned}
\frac{\partial^{2} \bar{\Phi}_{l}^{(1)}}{\partial \tau^{2}} & -\frac{2}{\tau} \frac{\partial \bar{\Phi}_{l}^{(1)}}{\partial \tau}-\frac{2}{r} \frac{\partial \bar{\Phi}_{l}^{(1)}}{\partial r}-\frac{\partial^{2} \bar{\Phi}_{l}^{(1)}}{\partial r^{2}}-\left[\frac{l(l+1)}{r^{2}}-\frac{M^{2}}{H^{2} \tau^{2}}\right] \bar{\Phi}_{l}^{(1)} \\
& =-4\left(\frac{\epsilon \tau}{r}\right)\left[\frac{\partial^{2} \bar{\Phi}_{l}^{(0)}}{\partial \tau^{2}}-\frac{1}{\tau} \frac{\partial \bar{\Phi}_{l}^{(0)}}{\partial \tau}-\frac{M^{2}}{2 H^{2} \tau^{2}} \bar{\Phi}_{l}^{(0)}\right] .
\end{aligned}
$$

The right hand side of 3.24 can be considered as a source term $J_{l}^{(1)}(r, \tau)$, of the form

$$
\begin{aligned}
J_{l}^{(1)}(r, \tau) & =-4\left(\frac{\epsilon \tau}{r}\right)\left[\frac{\partial^{2} \bar{\Phi}_{l}^{(0)}}{\partial \tau^{2}}-\frac{1}{\tau} \frac{\partial \bar{\Phi}_{l}^{(0)}}{\partial \tau}-\frac{M^{2}}{2 H^{2} \tau^{2}} \bar{\Phi}_{l}^{(0)}\right] \\
& =-4\left(\frac{\epsilon \tau}{r}\right) \int_{0}^{\infty} d k k^{2} j_{l}(k r)\left[\frac{\partial^{2} \bar{\Phi}_{k l}^{(0)}}{\partial \tau^{2}}-\frac{1}{\tau} \frac{\partial \bar{\Phi}_{k l}^{(0)}}{\partial \tau}-\frac{M^{2}}{2 H^{2} \tau^{2}} \bar{\Phi}_{k l}^{(0)}\right]
\end{aligned}
$$

To solve this equation we use the Green's function $G\left(r, \tau ; r^{\prime}, \tau^{\prime}\right)$

$$
G_{l}\left(r, \tau ; r^{\prime}, \tau^{\prime}\right)=\int_{0}^{\infty} d k k^{2} g_{k}\left(\tau, \tau^{\prime}\right) j_{l}(k r) j_{l}\left(k r^{\prime}\right),
$$

where $g_{k}$ satisfies the dynamics

$$
\frac{\partial^{2} g_{k}}{\partial \tau^{2}}-\frac{2}{\tau} \frac{\partial g_{k}}{\partial \tau}+\left[k^{2}+\frac{M^{2}}{H^{2} \tau^{2}}\right] g_{k}=\frac{2}{\pi} \delta\left(\tau-\tau^{\prime}\right) .
$$

The general solution for this equation is

$$
\begin{aligned}
g_{k}\left(\tau, \tau^{\prime}\right) & =(-\tau)^{3 / 2}\left\{A_{1} \mathcal{J}_{\nu}(-k \tau)+A_{2} \mathcal{Y}_{\nu}(-k \tau)-\left[\frac{\mathcal{J}_{\nu}(-k \tau)}{\left(-\tau^{\prime}\right)^{1 / 2}} \mathcal{Y}_{\nu}\left(-k \tau^{\prime}\right)\right.\right. \\
& \left.\left.-\frac{\mathcal{Y}_{\nu}(-k \tau)}{\left(-\tau^{\prime}\right)^{1 / 2}} \mathcal{J}_{\nu}\left(-k \tau^{\prime}\right)\right]\right\}
\end{aligned}
$$


where $0>\tau>\tau^{\prime}$. Hence, the field $\bar{\Phi}^{(1)}(r, \tau)$ can be expressed using the retarded Green function

$$
\bar{\Phi}_{l}^{(1)}(r, \tau)=\int_{0}^{\infty} d k k^{2} j_{l}(k r) \bar{\Phi}_{k l}^{(1)}(\tau)=\int^{\infty} d r^{\prime} r^{\prime 2} \int_{\tau_{i}}^{0} d \tau^{\prime} G_{l}\left(r, \tau ; r^{\prime}, \tau^{\prime}\right) J_{l}^{(1)}\left(r^{\prime}, \tau^{\prime}\right),
$$

where $\tau_{i}$ denotes the time when the source begins to operate and the modes $\bar{\Phi}_{k l}^{(1)}$ can be represented as

$$
\bar{\Phi}_{k l}^{(1)}(\tau)=\epsilon\left\{\alpha_{k l}(\tau) \bar{\Phi}_{k}^{(0)}(\tau)+\beta_{k l}(\tau)\left[\bar{\Phi}_{k}^{(0)}(\tau)\right]^{*}\right\}
$$

or explicitly

$$
\begin{aligned}
\bar{\Phi}_{k l}^{(1)}(\tau) & =\frac{e^{-i \nu \pi / 2} \sqrt{\pi} \epsilon}{2 H}(-\tau)^{3 / 2} \int_{\tau_{i}}^{\tau} d \tau^{\prime}\left\{A_{1} \mathcal{J}_{\nu}(-k \tau)+A_{2} \mathcal{Y}_{\nu}(-k \tau)-\left[\mathcal{J}_{\nu}(-k \tau) \mathcal{Y}_{\nu}\left(-k \tau^{\prime}\right)-\mathcal{Y}_{\nu}(-k \tau) \mathcal{J}_{\nu}\left(-k \tau^{\prime}\right)\right]\right\} \\
& \times \int_{0}^{\infty} \frac{d k^{\prime}}{\left(k^{\prime}\right)^{3}}\left\{\mathcal{H}_{\nu}^{(2)}\left(-k^{\prime} \tau^{\prime}\right)\left[H^{2}\left(3-4\left(\nu+\nu^{2}+\left(\tau^{\prime} k^{\prime}\right)^{2}\right)+2 M^{2}\right]-4 k^{\prime} H^{2} \tau^{\prime} \mathcal{H}_{\nu}^{(2)}\left(-k^{\prime} \tau^{\prime}\right)\right\}\right. \\
& \times \int_{0}^{\infty} d r^{\prime} r^{\prime} j_{l}\left(k r^{\prime}\right) j_{l}\left(k^{\prime} r^{\prime}\right)
\end{aligned}
$$

If we take into account the normalization conditions on the background modes $\bar{\Phi}_{k l}^{(0)}$, considered in (3.14), the expression (3.31) can be rewritten as

$$
\begin{aligned}
\bar{\Phi}_{k l}^{(1)}(\tau) & =\frac{\epsilon}{H^{2}} \int_{\tau_{i}}^{\tau} d \tau^{\prime}\left\{H^{2}\left[\bar{\Phi}_{k l}^{(0)}(-k \tau)\right]+\frac{i}{2} e^{-i \nu \pi / 2}\left[e^{-i \nu \pi / 2}\left[\bar{\Phi}_{k l}^{(0)}(-k \tau)\right]^{*} \mathcal{H}_{\nu}^{(2)}\left(-k \tau^{\prime}\right)\right.\right. \\
& \left.\left.-e^{i \nu \pi / 2}\left[\bar{\Phi}_{k l}^{(0)}(-k \tau)\right] \mathcal{H}_{\nu}^{(1)}\left(-k \tau^{\prime}\right)\right]\right\} \\
& \times \int_{0}^{\infty} d k^{\prime}\left(k^{\prime}\right)^{2}\left\{\mathcal { H } _ { \nu } ^ { ( 2 ) } ( - k ^ { \prime } \tau ^ { \prime } ) \left[H^{2}\left(3-4\left(\nu+\nu^{2}+\left(\tau^{\prime} k^{\prime}\right)^{2}\right)+2 M^{2}\right]\right.\right. \\
& \left.-4 k^{\prime} H^{2} \tau^{\prime} \mathcal{H}_{\nu+1}^{(2)}\left(-k^{\prime} \tau^{\prime}\right)\right\} \times \int_{0}^{\infty} d r^{\prime} r^{\prime} j_{l}\left(k r^{\prime}\right) j_{l}\left(k^{\prime} r^{\prime}\right)
\end{aligned}
$$

such that

$$
\begin{aligned}
\int_{0}^{1} d r^{\prime} r^{\prime} j_{l}\left(k r^{\prime}\right) j_{l}\left(k^{\prime} r^{\prime}\right) & =\frac{\pi}{2} \frac{\left(k^{\prime}\right)^{l} \Gamma(l+1)}{\Gamma(l+3 / 2) \Gamma(1 / 2)} \times F\left(l+1, \frac{1}{2} ; l+\frac{3}{2}, k^{\prime 2}\right), \\
\int_{1}^{\infty} d r^{\prime} r^{\prime} j_{l}\left(k r^{\prime}\right) j_{l}\left(k^{\prime} r^{\prime}\right) & =\frac{\pi}{2} \frac{\left(k^{\prime}\right)^{-(l+2)} \Gamma(l+1)}{\Gamma(l+3 / 2) \Gamma(1 / 2)} \times F\left(l+1, \frac{1}{2} ; l+\frac{3}{2}, \frac{1}{k^{\prime 2}}\right) .
\end{aligned}
$$

The expression (3.33) is valid for $k \in(0,1)$ and the expression (3.34) corresponds to the case $k \in(1, \infty)$. Furthermore, $F(a, b ; c, x)$ is the hypergeometric function. From eqs. (3.30) and (3.32), we obtain the following expressions for the coefficients $\alpha_{k l}$ and $\beta_{k l}$ :

$$
\begin{aligned}
\alpha_{k l}(\tau) & =\epsilon \int_{\tau_{i}}^{\tau} d \tau^{\prime}\left\{1-\frac{i}{2 H^{2}} \mathcal{H}_{\nu}^{(2)}\left(-k \tau^{\prime}\right)\right\} \int_{0}^{\infty} d k^{\prime}\left(k^{\prime}\right)^{2} \\
& \times\left\{\mathcal{H}_{\nu}^{(2)}\left(-k^{\prime} \tau^{\prime}\right)\left[H^{2}\left(3-4\left(\nu+\nu^{2}+\left(\tau^{\prime} k^{\prime}\right)^{2}\right)+2 M^{2}\right]-4 k^{\prime} H^{2} \tau^{\prime} \mathcal{H}_{\nu+1}^{(2)}\left(-k^{\prime} \tau^{\prime}\right)\right\}\right. \\
& \times \int_{0}^{\infty} d r^{\prime} r^{\prime} j_{l}\left(k r^{\prime}\right) j_{l}\left(k^{\prime} r^{\prime}\right) \\
\beta_{k l}(\tau) & =\frac{i \epsilon e^{-i \nu \pi}}{2 H^{2}} \int_{\tau_{i}}^{\tau} d \tau^{\prime} \mathcal{H}_{\nu}^{(1)}\left(-k \tau^{\prime}\right) \int_{0}^{\infty} d k^{\prime}\left(k^{\prime}\right)^{2} \\
& \times\left\{\mathcal{H}_{\nu}^{(2)}\left(-k^{\prime} \tau^{\prime}\right)\left[H^{2}\left(3-4\left(\nu+\nu^{2}+\left(\tau^{\prime} k^{\prime}\right)^{2}\right)+2 M^{2}\right]-4 k^{\prime} H^{2} \tau^{\prime} \mathcal{H}_{\nu+1}^{(2)}\left(-k^{\prime} \tau^{\prime}\right)\right\}\right. \\
& \times \int_{0}^{\infty} d r^{\prime} r^{\prime} j_{l}\left(k r^{\prime}\right) j_{l}\left(k^{\prime} r^{\prime}\right)
\end{aligned}
$$


where $\tau_{i}=-1 / H=-\psi_{0}$ is the conformal time when inflation starts, which will be considered when $a\left(\tau_{i}\right)=1$. The wavenumbers related to the horizons $k_{H}$ and $k_{S c h}$, are given respectively by the equations (3.18) and (3.22) and $k_{g a}$ is the gravitational-antigravitational wavenumber

$$
k_{g a}=\frac{2 \pi}{a(\tau) r_{g a}}\left[\frac{2 a(\tau) r_{g a}}{2 a(\tau) r_{g a}+G m}\right]^{2},
$$

where $r_{g a}$ is given by (2.17). Furthermore, the power spectrum for the $\Phi^{(1)}$-fluctuations is [see eqs. (3.8) and (3.9)]

$$
\mathcal{P}_{k l}^{(1)}=\frac{H^{2}}{4 \pi^{2}} \epsilon \Delta_{k l}^{(1)}(\tau)
$$

where $\Delta_{k l}^{(1)}(\tau)$ is given by

$$
\Delta_{k l}^{(1)}(\tau)=\frac{2 \pi k^{5}}{\epsilon H^{2}} \operatorname{Re}\left\{\Phi_{k l}^{(1)}(\tau)\left(\Phi_{k l}^{(0)}(\tau)\right)^{*}\right\}=\frac{2 \pi k^{5}}{H^{2}}\left\{\left|\Phi_{k l}^{(0)}\right|^{2} \operatorname{Re}\left[\alpha_{k l}(\tau)\right]+\operatorname{Re}\left[\beta_{k l}^{*}\left(\Phi_{k l}^{(0)}\right)^{2}\right]\right\},
$$

where we have used the expression (3.30). In the following subsections we shall calculate the spectrums $\Delta_{k l}^{(1)}(\tau)$ on scales where gravity is repulsive (big scales, of today cosmological scales) and attractive (small scales, or today astrophysical scales) in the first order approximation.

\subsubsection{First order: power spectrum on cosmological scales}

Now we consider the spectrum (3.39). To calculate $\alpha_{k l}(\tau)$ and $\beta_{k l}(\tau)$, we shall make use of the asymptotic expressions for the first and second kind Hankel functions: $\mathcal{H}_{\nu}^{(1,2)}[x] \simeq \mp \frac{i}{\pi} \Gamma(\nu)(x / 2)^{-2}$ [in our case $x(\tau)=-k \tau \ll 1]$, in (3.35) and (3.36)

$$
\begin{aligned}
\left.\alpha_{k l}(\tau)\right|_{-k \tau \ll 1} & \simeq \frac{i \epsilon \pi}{2} \frac{\Gamma(l+1)}{\Gamma(l+3 / 2) \Gamma(1 / 2)} \int_{k_{H}}^{k_{g a}} d k^{\prime} \int_{\tau_{i}}^{\tau} d \tau^{\prime}\left\{1+\frac{2^{\nu-1} k^{-\nu} \Gamma(\nu)\left(-\tau^{\prime}\right)^{-2 \nu}}{\pi H^{2}}\right\}\left(k^{\prime}\right)^{2-\nu+l} \\
& \times\left\{2 ^ { \nu } \frac { \Gamma ( \nu ) } { \pi } \left[H^{2}\left[3-4\left(\nu+\nu^{2}+\left(k^{\prime} \tau^{\prime}\right)^{2}+2 M^{2}\right]\right]\right.\right. \\
& \left.-\frac{k^{\prime} \tau^{\prime}}{\pi} H^{2} \Gamma(\nu+1)\left(k^{\prime}\right)^{-(\nu+1)} 2^{\nu+3}\left(-\tau^{\prime}\right)^{-(\nu+1)}\right\} \times_{2} F_{1}\left[l+1,1 / 2 ; l+3 / 2 ;\left(k^{\prime}\right)^{2}\right] \\
\left.\beta_{k l}(\tau)\right|_{-k \tau \ll 1} & \simeq i \frac{\epsilon \Gamma(l+1) \Gamma^{2}(\nu) e^{-i \nu \pi}}{4 \pi H^{2} \Gamma(l+3 / 2) \Gamma(1 / 2)} \int_{\tau_{i}}^{\tau} d \tau^{\prime}\left(-\frac{k \tau^{\prime}}{2}\right)^{-\nu} \\
& \times \int_{k_{H}}^{k_{g a}} d k^{\prime}\left(k^{\prime}\right)^{l}{ }_{2} F_{1}\left[l+1,1 / 2 ; l+3 / 2 ;\left(k^{\prime}\right)^{2}\right] \\
& \times\left\{\left[H^{2}\left[3-4\left(\nu+\nu^{2}+\left(\tau^{\prime} k^{\prime}\right)^{2}\right]+2 M^{2}\right]-4 i \frac{k^{\prime} \tau^{\prime} H^{2} \Gamma(\nu+1)}{\pi}\left(-\frac{k^{\prime} \tau}{2}\right)^{-(\nu+1)}\right\}\right.
\end{aligned}
$$

where ${ }_{2} F_{1}[a, b ; c ; y(k)]$ is the hypergemetric function. When the horizon entry we obtain the following approximated spectrum on cosmological scales:

$$
\left.\Delta_{k l}^{(1)}\left(\tau_{e}\right)\right|_{-k \tau \ll 1} \simeq 2 \operatorname{Re}\left[\left.\alpha_{k l}\left(\tau_{e}\right)\right|_{-k \tau \ll 1}-\left.\beta_{k l}\left(\tau_{e}\right)\right|_{-k \tau \ll 1}\right],
$$

where $\left.\alpha_{k l}\left(\tau_{e}\right)\right|_{-k \tau \ll 1}$ and $\left.\beta_{k l}\left(\tau_{e}\right)\right|_{-k \tau \ll 1}$ are the expressions (3.40) and (3.41) when $\tau \rightarrow \tau_{e}$, with $1 /\left(-\tau_{e}\right)>H e^{60}$. In other words $\left(-\tau_{e}\right)$ is sufficiently close to zero to preserve the required large 
scale flatness of the universe at the end of inflation. In this limit case the coefficients are

$$
\begin{aligned}
\left.\alpha_{k l}\left(\tau_{e}\right)\right|_{-k \tau \ll 1} & \simeq \frac{i \epsilon \pi}{2} \frac{\Gamma(l+1)}{\Gamma(l+3 / 2) \Gamma(1 / 2)} \int_{k_{H}}^{k_{g a}} d k^{\prime} \int_{\tau_{i}}^{\tau_{e}} d \tau^{\prime}\left\{1+\frac{2^{\nu-1} k^{-\nu} \Gamma(\nu)\left(-\tau^{\prime}\right)^{-2 \nu}}{\pi H^{2}}\right\}\left(k^{\prime}\right)^{2-\nu+l} \\
& \times\left\{2^{\nu} \frac{\Gamma(\nu)}{\pi}\left[H^{2}\left[3-4\left(\nu+\nu^{2}+\left(k^{\prime} \tau^{\prime}\right)^{2}+2 M^{2}\right]\right]-\frac{k^{\prime} \tau^{\prime}}{\pi} H^{2} \Gamma(\nu+1)\left(k^{\prime}\right)^{-(\nu+1)} 2^{\nu+3}\left(-\tau^{\prime}\right)^{-(\nu+1)}\right\}\right. \\
& \times{ }_{2} F_{1}\left[l+1,1 / 2 ; l+3 / 2 ;\left(k^{\prime}\right)^{2}\right], \\
\left.\beta_{k l}\left(\tau_{e}\right)\right|_{-k \tau \ll 1} & \simeq i \frac{\epsilon \Gamma(l+1) \Gamma^{2}(\nu) e^{-i \nu \pi}}{4 \pi H^{2} \Gamma(l+3 / 2) \Gamma(1 / 2)} \int_{\tau_{i}}^{\tau_{e}} d \tau^{\prime}\left(-\frac{k \tau^{\prime}}{2}\right)^{-\nu} \int_{k_{H}}^{k_{g a}} d k^{\prime}\left(k^{\prime}\right)^{l}{ }_{2} F_{1}\left[l+1,1 / 2 ; l+3 / 2 ;\left(k^{\prime}\right)^{2}\right] \\
& \times\left\{\left[H^{2}\left[3-4\left(\nu+\nu^{2}+\left(\tau^{\prime} k^{\prime}\right)^{2}\right]+2 M^{2}\right]-4 i \frac{k^{\prime} \tau^{\prime} H^{2} \Gamma(\nu+1)}{\pi}\left(-\frac{k^{\prime} \tau^{\prime}}{2}\right)^{-(\nu+1)}\right\} .\right.
\end{aligned}
$$

Since

$$
2 \operatorname{Re}\left[\left.\alpha_{k l}\left(\tau_{e}\right)\right|_{-k \tau \ll 1}\right]=0,
$$

the function

$$
\begin{aligned}
\left.\Delta_{k l}^{(1)}\left(\tau_{e}\right)\right|_{-k \tau \ll 1} & \simeq \frac{\epsilon 2^{2(\nu+1)} \Gamma(l+1) \Gamma^{2}(\nu) \Gamma(\nu+1)}{\pi^{2} \Gamma(l+3 / 2) \Gamma(1 / 2)} k^{-\nu} \\
& \times \int_{\tau_{i}}^{\tau_{e}} d \tau^{\prime}\left(-\tau^{\prime}\right)^{-2 \nu} \int_{k_{H}}^{k_{g a}} d k^{\prime}\left(k^{\prime}\right)^{l-\nu}{ }_{2} F_{1}\left[l+1,1 / 2 ; l+3 / 2 ;\left(k^{\prime}\right)^{2}\right],
\end{aligned}
$$

is determined by real part of (3.44) which describes the spectrum of the scalar field fluctuations at the end of inflation in the infrared region, in which gravity is repulsive. This spectrum is enclosed by the wavenumbers $k_{g a}$ and $k_{H}$, related respectively to the gravitational-antigravitational radius and the Hubble horizon. Notice that this spectrum is linear with $\epsilon=m H / M_{p}^{2}$, so that its amplitude depends on the BH mass $m$. In absence of the BH (i.e., for $m=0$ ), $\Delta_{k l}^{(1)}=0$.

\subsubsection{First order: power spectrum on small scales}

To calculate the spectrum at first order on small scales, we shall make use of the asymptotic expressions for big argument Hankel functions: $\mathcal{H}_{\nu}^{(1,2)}[x(\tau)] \simeq(2 /(\pi x))^{1 / 2} e^{ \pm i[x(\tau)-\nu \pi / 2-\pi / 4]}$, where $x(\tau)=-k \tau$. The coefficients $\alpha_{k l}(\tau)$ and $\beta_{k l}(\tau)$ are given by

$$
\begin{aligned}
\left.\alpha_{k l}(\tau)\right|_{-k \tau \gg 1} & \simeq \epsilon \sqrt{\frac{\pi}{2}} \frac{\Gamma(l+1)}{\Gamma(l+3 / 2) \Gamma(1 / 2)} \int_{\tau_{i}}^{\tau}\left\{1-\frac{i}{H^{2}} \sqrt{\frac{2}{\pi}}\left(-k \tau^{\prime}\right)^{-1 / 2} e^{-i\left[-k \tau^{\prime}-\nu \pi / 2-\pi / 4\right]}\right\} \\
& \times \int_{k_{g a}}^{k_{S c h}}\left(k^{\prime}\right)^{-l}{ }_{2} F_{1}\left[l+1,1 / 2 ; l+3 / 2 ; 1 /\left(k^{\prime}\right)^{2}\right]\left\{\left(-k^{\prime} \tau^{\prime}\right)^{-1 / 2} e^{-i\left[-k^{\prime} \tau^{\prime}-\nu \pi / 2-\pi / 4\right]}\right. \\
& \times\left[H^{2}\left[3-4\left(\nu+\nu^{2}+\left(\tau^{\prime} k^{\prime}\right)^{2}\right]+2 M^{2}\right]-4 k^{\prime} \tau^{\prime} H^{2}\left(-k^{\prime} \tau^{\prime}\right)^{-1 / 2} e^{-i\left[-k^{\prime} \tau^{\prime}-(\nu+1) \pi / 2-\pi / 4\right]}\right\}, \\
\left.\beta_{k l}(\tau)\right|_{-k \tau \gg 1} & \simeq \frac{i \epsilon}{2 H^{2}} \frac{\Gamma(l+1)}{\Gamma(l+3 / 2) \Gamma(1 / 2)} e^{-i \nu \pi} \int_{\tau_{i}}^{\tau} d \tau^{\prime}\left(-k \tau^{\prime}\right)^{-1 / 2} e^{-i\left[-k \tau^{\prime}-\nu \pi / 2-\pi / 4\right]} \int_{k_{g a}}^{k_{S c h}} d k^{\prime}\left(k^{\prime}\right)^{-l} \\
& \times\left\{( - k ^ { \prime } \tau ^ { \prime } ) ^ { - 1 / 2 } e ^ { - i [ - k ^ { \prime } \tau ^ { \prime } - \nu \pi / 2 - \pi / 4 ] } \left[H^{2}\left[3-4\left(\nu+\nu^{2}+\left(\tau^{\prime} k^{\prime}\right)^{2}\right]+2 M^{2}\right]\right.\right. \\
& \left.-4 \sqrt{\frac{2}{\pi}} H^{2} k^{\prime} \tau^{\prime}\left(-k^{\prime} \tau^{\prime}\right)^{-1 / 2} e^{-i\left[-k^{\prime} \tau^{\prime}-(\nu+1) \pi / 2-\pi / 4\right]}\right\}{ }_{2} F_{1}\left[l+1,1 / 2 ; l+3 / 2 ; 1 /\left(k^{\prime}\right)^{2}\right] .
\end{aligned}
$$


The function $\left.\Delta_{k l}^{(1)}\left(\tau_{e}\right)\right|_{-k \tau \gg 1}$ describes the spectrum of the scalar field fluctuations on scales between the the Schwarzschild horizon and gravitational-antigravitational distances, which are related to a wavenumber $k_{g a}$, and describes the region of the spectrum where gravity is attractive. At the end of inflation $\left.\Delta_{k l}^{(1)}\left(\tau_{e}\right)\right|_{-k \tau \gg 1}$ is given by

$$
\left.\Delta_{k l}^{(1)}\left(\tau_{e}\right)\right|_{-k \tau \gg 1}=\frac{2 \pi k^{5}}{H^{2}}\left\{\left|\Phi_{k l}^{(0)}\right|_{-k \tau \gg 1}^{2} \operatorname{Re}\left[\left.\alpha_{k l}\left(\tau_{e}\right)\right|_{-k \tau \gg 1}\right]+\operatorname{Re}\left[\left.\beta_{k l}\left(\tau_{e}\right)\right|_{-k \tau \gg 1}\left(\Phi_{k l}^{(0)}\right)_{-k \tau \gg 1}^{2}\right]\right\},
$$

where

$$
\begin{aligned}
\left.\alpha_{k l}\left(\tau_{e}\right)\right|_{-k \tau \gg 1} & \simeq \epsilon \sqrt{\frac{\pi}{2}} \frac{\Gamma(l+1)}{\Gamma(l+3 / 2) \Gamma(1 / 2)} \int_{\tau_{i}}^{\tau_{e}}\left\{1-\frac{i}{H^{2}} \sqrt{\frac{2}{\pi}}\left(-k \tau^{\prime}\right)^{-1 / 2} e^{-i\left[-k \tau^{\prime}-\nu \pi / 2-\pi / 4\right]}\right\} \\
& \times \int_{k_{g a}}^{k_{S c h}}\left(k^{\prime}\right)^{-l}{ }_{2} F_{1}\left[l+1,1 / 2 ; l+3 / 2 ; 1 /\left(k^{\prime}\right)^{2}\right]\left\{\left(-k^{\prime} \tau^{\prime}\right)^{-1 / 2} e^{-i\left[-k^{\prime} \tau^{\prime}-\nu \pi / 2-\pi / 4\right]}\right. \\
& \times\left[H^{2}\left[3-4\left(\nu+\nu^{2}+\left(\tau^{\prime} k^{\prime}\right)^{2}\right]+2 M^{2}\right]-4 k^{\prime} \tau^{\prime} H^{2}\left(-k^{\prime} \tau^{\prime}\right)^{-1 / 2} e^{-i\left[-k^{\prime} \tau^{\prime}-(\nu+1) \pi / 2-\pi / 4\right]}\right\}, \\
\left.\beta_{k l}\left(\tau_{e}\right)\right|_{-k \tau \gg 1} & \simeq \frac{i \epsilon}{2 H^{2}} \frac{\Gamma(l+1)}{\Gamma(l+3 / 2) \Gamma(1 / 2)} e^{-i \nu \pi} \int_{\tau_{i}}^{\tau_{e}} d \tau^{\prime}\left(-k \tau^{\prime}\right)^{-1 / 2} e^{-i\left[-k \tau^{\prime}-\nu \pi / 2-\pi / 4\right]} \int_{k_{g a}}^{k_{S c h}} d k^{\prime}\left(k^{\prime}\right)^{-l} \\
& \times\left\{( - k ^ { \prime } \tau ^ { \prime } ) ^ { - 1 / 2 } e ^ { - i [ - k ^ { \prime } \tau ^ { \prime } - \nu \pi / 2 - \pi / 4 ] } \left[H^{2}\left[3-4\left(\nu+\nu^{2}+\left(\tau^{\prime} k^{\prime}\right)^{2}\right]+2 M^{2}\right]\right.\right. \\
& \left.-4 \sqrt{\frac{2}{\pi}} H^{2} k^{\prime} \tau^{\prime}\left(-k^{\prime} \tau^{\prime}\right)^{-1 / 2} e^{-i\left[-k^{\prime} \tau^{\prime}-(\nu+1) \pi / 2-\pi / 4\right]}\right\}{ }_{2} F_{1}\left[l+1,1 / 2 ; l+3 / 2 ; 1 /\left(k^{\prime}\right)^{2}\right], \\
\left|\Phi_{k l}^{(0)}\right|_{-k \tau \gg 1}^{2} & \simeq \frac{H^{2}}{2 \pi} \frac{(-\tau)^{2}}{k}, \\
\left(\Phi_{k l}^{(0)}\right)_{-k \tau \gg 1}^{2} & \simeq \frac{H^{2}}{2 \pi} \frac{(-\tau)^{2}}{k} e^{-2 i[(-k \tau)-\pi / 4]},
\end{aligned}
$$

which also becomes zero in absence of the BH. The calculation of $\left.\Delta_{k l}^{(1)}\left(\tau_{e}\right)\right|_{-k \tau \gg 1}$ is very complicated, but one can see clearly using the expansion for the hypergeometric function:

$$
{ }_{2} F_{1}(a, b ; c ; z)=\frac{\Gamma(c)}{\Gamma(a) \Gamma(b)} \sum_{n=0}^{\infty} \frac{\Gamma(a+n) \Gamma(b+n)}{\Gamma(c+n)} \frac{z^{n}}{n !},
$$

that terms of $k^{\prime}$ with positive potentia are relevant for large values of $l$, i.e., for smaller scales.

\section{Final comments}

In this letter we have studied inflationary quantum scalar field fluctuations of an effective 4D scalar field, in the framework of an extended version of general relativity derived from a $5 \mathrm{D}$ vacuum theory of gravity. The interesting aspect of this extended version of general relativity, is that gravity manifests itself as repulsive at large (cosmological) scales and attractive at short scales 14. We assume a 5D spacetime described geometrically by the SdS metric (2.1), endowed with a 5D massless scalar field $\varphi$. In order to consider an inflationary cosmological setting we write the metric in (2.1) in the dynamical coordinate chart $(\tau, r, \theta, \phi)$. In this new coordinates the length scale that separates regions on which gravity changes from attractive to repulsive, named the gravity-antigravity radius $r_{g a}$, becomes dynamical and it varies inversely proportional to the conformal scale factor $a(\tau)$, as 
it was shown in the expression (2.17). This can be interpreted on $4 \mathrm{D}$ cosmological settings as when the universe expands, the region where gravity is repulsive becomes larger every time. When we go down from five to four dimensions via a foliation of the 5D spacetime in the fifth coordinate, it is a well known result that the 5D massless scalar field $\varphi$ can be seen in 4D as a massive effective scalar field, as it is shown in this particular case for $\bar{\varphi}(\tau, r)$ by the equation (2.14). This is an important characteristic of $5 \mathrm{D}$ free scalar fields were they move on an effective $4 \mathrm{D}$ hypersurface obtained by a static foliaton [in our case $\psi=\psi_{0}=c^{2} / H=m / \zeta$ ].

We quantized the $4 \mathrm{D}$ effective scalar field $\bar{\varphi}$ following the canonical procedure and we found that its quantum modes evolve according to the equation (3.5). Given the difficulty of finding exact solutions for the modes equation (3.5), we decided to use an expansion for the modes $\bar{\Phi}(\tau, r)$ in powers of a small parameter $\epsilon=m H / M_{p}^{2}$. The fact that $\epsilon \leq 0.353553$ is a small parameter arises naturally from the model and provides a constraint for the extra coordinate: $\psi_{0} \geq(2 \sqrt{2} m) / M_{p}^{2}$. From our analysis we obtained that it is possible to have exact solutions $\bar{\Phi}$ at least at zeroth and first orders in the expansion. Finally, one important result obtained in this model is that at the end of an stage of de-Sitter inflation, the corresponding spectrum of fluctuations

- at zeroth order results to be independent of the scalar field mass $M$ on Schwarzschild scales,

- while on cosmological scales it exhibits a mass $M$ dependence through the parameter $\nu^{2}=$ $\frac{9}{4}-\frac{M^{2}}{H^{2}}$, because the spectrum go as $k^{3-2 \nu}$.

At first order of expansion, we found that on both length scales the spectrum depends on the inflaton field mass $M$ and the amplitude depends on the BH mass $m$, which is the source of spatial inhomogeneity in our model.

\section{Acknowledgements}

J.E.M.A acknowledges CONACYT (México) and M.B. acknowledges UNMdP and CONICET (Argentina) for financial support.

\section{References}

[1] J. Einasto, et. al, Astroph. J. 519: 441 (1999).

[2] J. M. Overduin and P. S. Wesson, Phys. Rept. 283: 303 (1997); P.S. Wesson, Space-Time-Matter, World Scientific, Singapore (1999).

[3] Roy Maartens, Livng Rev. Rel. 7, 7 (2004).

[4] G. Nordström, Phys. Z. 15, 504 (1914).

[5] T. Kaluza, Sitz. Preuss. Akad. Wiss. 33, 996 (1921); O. Klein, Phys. Z 37 895, (1926).

[6] M. Pavsic, The Landscape of Theoretical Physics: A Global View- From Point Particles to the Brane World and Beyond in the Search of a Unifying Principle of Physics. Springer Heidelberg (2002).

[7] P. S. Wesson, Gen. Rel. Grav. 16: 193 (1984); P. Wesson, Gen. Rel. Grav. 22: 707 (1990); P. S. Wesson, Phys. Lett. B276: 299 (1992); P. S. Wesson and J. Ponce de Leon, J. Math. Phys. 33: 3883 (1992); H. Liu and P. S. Wesson, J. Math. Phys. 33: 3888 (1992); P. Wesson, H. Liu and P. Lim, Phys. Lett. B298: 69 (1993).

[8] J. Ponce de Leon, Mod. Phys. Lett. A16, 2291-2304, (2001).

[9] P. S. Wesson, J. Ponce de Leon, J. Math. Phys. 33: 3883 (1992).

[10] C. Romero, R. Tavakol and R. Zalaletdinov, Gen. Rel. Grav. 28: 365 (1996). 
[11] H. Y. Liu and B. Mashhoon, Phys. Lett. A272: 26 (2000);

B. Mashhoon and P. S. Wesson, Class. Quant. Grav. 21: 3611 (2004).

[12] M. L. Liu, H. Y. Liu, L. X. Xu and P. S. Wesson, Mod. Phys. Lett. A21: 39 (2006).

[13] L. Randall and R. Sundrum, Phys. Rev. Lett. 83: 3370 (1999);

L. Randall and R. Sundrum, Phys. Rev. Lett. 83: 4690 (1999).

[14] J. E. Madriz Aguilar and M. Bellini, Phys. Lett. B679: 306 (2009).

[15] T. Shiromizu, D. Ida and T, Torii, J. High Energy Physics 11: 010 (2001).

[16] W. Rindler, Essential Relativity. (2nd. edition) Springer, Berlin, (1977);

S. S. Seahra and P. S. Wesson, Gen. Rel. Grav. 33, 1731 (2001);

P. S. Wesson, Five-dimensional Physics, Classical and Quantum Consequences of Kaluza-Klein Cosmology, World Scientific, New Jersey (2006).

[17] M. Bellini, Nucl. Phys. B660: 389 (2003).

[18] H. T. Cho, K. W. Ng and I, C. Wang, Scalar field fluctuations in Schwarzschild-de Sitter space-time. E-print arXiv: 0905.2041. 